\title{
First thoracic vertebral body as reference for endotracheal tube placement
}

\author{
Marc P Blayney, Donald R Logan
}

\begin{abstract}
After intubation in newborn infants, the placement of the endotracheal tube in the trachea must be checked by a chest radiograph. The most commonly used reference point for placement is the medial ends of the clavicles. The position of the clavicles may be variable. The present study was carried out to determine whether the body of the first thoracic vertebra should be used instead of the clavicles because its position on chest radiographs is more constant. Seventy eight radiographs obtained from 35 neonates were examined prospectively. The carina was situated between $T 3$ and T5, most commonly at T3-4 or T4 (85\%). The position of the clavicles varied markedly from patient to patient and within the same patient on different days, and this variation was significantly higher than that of the carina. On $65(83 \%)$ examinations the clavicles lay above the first thoracic vertebra.

It is recommended that, for accurate placement within the trachea, the tip of the endotracheal tube should be placed at the level of the body of the first thoracic vertebra; this could be used as the sole reference point on chest radiographs obtained in the neonatal period.

(Arch Dis Child 1994; 71: F32-F35)
\end{abstract}

After intubation in newborn infants, the position of the endotracheal tube in the trachea must be checked to ensure a minimum number of complications such as right main bronchus intubation (which can lead to atelectasis and hypoxia) and high placement (accidental extubation). Methods used to determine accurate positioning within the trachea have included digital palpation, ${ }^{1}$ fibre optics, ${ }^{2}$ ultrasound, ${ }^{3}$ and, more recently, a metal ring embedded in the endotracheal tube. ${ }^{4}$

Chest radiographs remain the method of choice, however, though guidelines for interpretation vary. The lower limit for the position of the endotracheal tube is usually measured in relation to the carina. The tip of the endotracheal tube should be between 0.2 $\mathrm{cm}^{5}$ and $2.0 \mathrm{~cm}^{6}$ above the carina. There are times when the carina is not clearly visible on the radiograph and as these measurements do not take the size of the infant into account, the carina is a less than ideal reference point.

A commonly used upper limit is the medial border of the clavicles. ${ }^{7}$ During one trial, ${ }^{4}$ however, because the position of the clavicles was noted to be variable, the body of the first thoracic vertebra (T1) was used, as this is the posterior marker of the thoracic inlet. ${ }^{8} \mathrm{We}$ have not been able to find any clinical studies which have confirmed the work of Bednarek and Kuhns, who found that the medial ends of the clavicles were a good landmark of the midtrachea on a chest radiograph. ${ }^{1}$

The purpose of this study was firstly to evaluate the position of the clavicles on chest radiographs in relation to the body of the first thoracic vertebra (T1) and, secondly, to evaluate the relation of both to the carina to determine which is a more constant and, hence, a more reliable reference point.

\section{Methods}

Chest radiographs were obtained after endotracheal tube intubation to verify the position of the endotracheal tube. All radiographs were obtained on a portable machine in the neonatal intensive care unit. The radiographs were reviewed independently by the two authors and the following data were recorded: (a) the positions of the medial ends of the clavicles and the carina in relation to the cervical or thoracic vertebra, or intervertebral disc space; and (b) the distances in $\mathrm{cm}$, using a ruler on the films, from the carina to (i) the clavicles (midpoint of medial ends) and (ii) T1 (midpoint of body).

All the radiographs were anteroposterior films of the chest and were taken at peak inspiration with the infant lying supine, at a tube-film distance of $101 \mathrm{~cm}$ (40 in), with the beam perpendicular to the sternum and aimed at the sternal angle. The infant's head was maintained in the midline and the neck was in the neutral position. This standardised technique was used to eliminate the effects of magnification.

The vertebrae were numbered by counting the number of ribs present, starting superiorly by tracing the first rib and proceeding inferiorly to identify the 12 th rib, if present, and thus T12. The results were subsequently reviewed by the two authors and the radiographs were re-examined if a major discrepancy was noted with regard to numbering of the vertebral bodies or the measured distance. The distances were remeasured if there was more than a $0.2 \mathrm{~cm}$ difference.

A limit of four chest radiographs for each patient was set to avoid bias towards the smaller infants who require more frequent radiographs. Only chest radiographs performed within the first 14 days of life were used, thus minimising the risk of wider intrapatient variations due to changes in the infants' weight and size. 


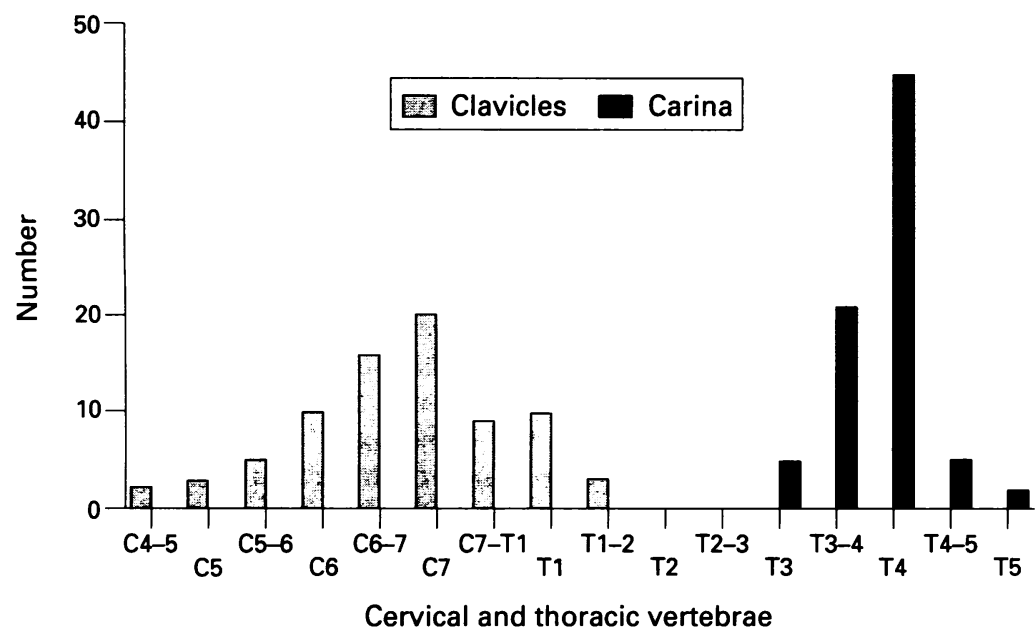

Figure 1 Position of the clavicles and carina in relation to the vertebral bodies or intervertebral disc spaces. The $x$ axis represents the cervical and thoracic vertebral bodies and their intervertebral disc spaces; the $y$ axis represents the number of occasions each point was noted on chest radiograph. The total number of chest radiographs was 78 .

\section{EXCLUSIONS}

Chest radiographs in which the position of the carina could not be determined were excluded, as were those which did not include the clavicles. Single chest and abdomen radiographs were also excluded.

\section{STATISTICS}

Variations in the positions of the medial ends of the clavicles and of the carina were partitioned into two types - that is, among

Table 1 Distances from the carina to the medial ends of the clavicles and to the body of $T 1$

\begin{tabular}{|c|c|c|c|}
\hline \multirow{2}{*}{$\begin{array}{l}\text { Birth weight } \\
\text { (g) }\end{array}$} & \multirow{2}{*}{$\begin{array}{l}\text { No of } \\
\text { chest } \\
\text { radiographs }\end{array}$} & \multicolumn{2}{|l|}{ Distance $(\mathrm{cm})^{\star}$} \\
\hline & & Carina to clavicles & Carina to $T 1$ \\
\hline $\begin{array}{r}500-1000 \\
1001-1500 \\
1501-2000 \\
2001-2500 \\
2501-3000 \\
3001-3500 \\
3501-4000\end{array}$ & $\begin{array}{r}27 \\
19 \\
13 \\
10 \\
4 \\
4 \\
1\end{array}$ & $\begin{array}{l}1.7(0.38) \\
2.0(0.29) \\
2 \cdot 2(0.34) \\
2.6(0.41) \\
3.1(0.16) \\
3.0(0.5) \\
3.3\end{array}$ & $\begin{array}{l}1.4(0.14) \\
1.5(0.19) \\
1.5(0.16) \\
1.6(0.26) \\
1.7(0.24) \\
1.8(0.10) \\
1.7\end{array}$ \\
\hline
\end{tabular}

$\star$ Distances are the mean (SD) for all values in the weight range.

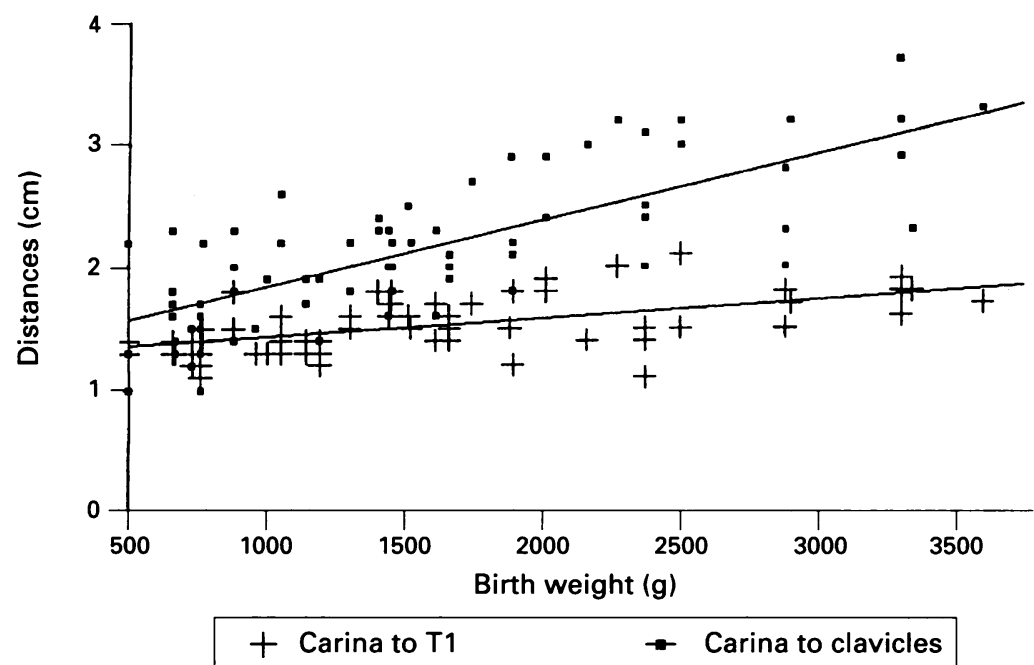

Figure 2 Regression lines for the distances $(\mathrm{cm})$ from the carina to the first thoracic vertebrae and the medial ends of the clavicles. The $x$ axis is birth weight $(g)$; the $y$ axis is distance $(\mathrm{cm})$. Points may represent more than one measurement. patient variation and within patient variation by the one way analysis of variance (ANOVA). They were then correspondingly compared using $\mathrm{F}$ tests. Regression analysis was applied to relate the infants' birth weight and gestation to the distance from the carina to the medial ends of the clavicle and the body of $T 1$.

Data processing and statistical analyses were performed by the PC SAS statistical software. ${ }^{9}$

\section{Results}

We examined 84 chest radiographs from 37 infants, but excluded six studies and two infants, giving a total of 78 radiographs. Three were excluded because the carina could not be seen, two were combined abdomen and chest films, and one was excluded because of severe flexion of the neck. Twelve infants had only one chest radiograph, seven had two, 12 had three, and four had four chest radiographs examined. The infants weighed from 500 to $3600 \mathrm{~g}$ at birth (mean (SD) $1642(825) \mathrm{g}$ ) and the gestational age ranged from 24 to 40 weeks (mean (SD) $30.3(4.5)$ weeks). The principle reason for intubation was respiratory distress syndrome.

Figure 1 gives the positions of the medial ends of the clavicles and the carina. On two occasions the clavicles were overlying the C4-5 intervertebral disc space and the lowest recorded position was the T1-2 intervertebral disc space. On $65(83 \%)$ examinations the clavicles lay above $\mathrm{T} 1$; their positions coincided on $10(13 \%)$ occasions. The total range was four vertebrae and their disc spaces. The position of the carina ranged from the third to the fifth thoracic vertebral disc (two vertebrae and their disc spaces) and in $66(85 \%)$ of the radiographs the carina was at either the T3-4 disc space or at T4.

Table 1 gives the distances from the carina to the medial ends of the clavicles and to the body of T1. Figure 2 shows the regression lines for these distances plotted against birth weight.

\section{STATISTICS}

The variations of the positions of the clavicles and carina were assessed using $F$ tests and a coding system which assigned one point to each disc to intervertebral disc space, with $\mathrm{T} 1$ as point 0 and ranging from -7 for the $\mathrm{C} 4-5$ disc space to +8 for the $\mathrm{T} 5$ disc. The position of the clavicles varied markedly from patient to patient and within the same patient on different days. ANOVA results (table 2) indicate that the position of the clavicles is determined with significantly higher variation than the position of the carina between different patients and within individual patients. It

Table 2 Results of ANOVA: variation of the position of clavicles and carina

\begin{tabular}{lllll}
\hline $\begin{array}{l}\text { Source of } \\
\text { variation }\end{array}$ & $\begin{array}{l}\text { Degrees of } \\
\text { freedom }\end{array}$ & Clavicle & Carina & $\begin{array}{l}F \\
\text { test }\end{array}$ \\
\hline Among patients & 34 & $172 \cdot 21$ & $31 \cdot 63$ & 5.45 \\
Within patients & 43 & 87.08 & $16 \cdot 17$ & $5 \cdot 39$ \\
Total & 77 & $259 \cdot 29$ & $47 \cdot 80$ & \\
\hline
\end{tabular}


should be noted that the values of the $F$ tests are invariant under any linear transformation or scale change of the coding system.

\section{Discussion}

Our results show that the position of the medial ends of the clavicles on chest radiographs can vary within a group of newborn infants and in the same patient at different times. The variability ranged from zero to two and a half intervertebral disc spaces within the group of 23 patients who had two or more chest radiographs (average $1 \cdot 15$ intervertebral disc spaces). In the complete group of 35 infants, the clavicles ranged in position from C4-5 (the level of the vocal cords) to T1-2, a total of four intervertebral disc spaces. The seventh cervical vertebra was the most common level for the clavicles $(24 \%)$ rather than $\mathrm{T} 1(13 \%)$, as might have been expected if the clavicles are the anterior markers of the thoracic inlet. Our findings differ from Bednarek and Kuhns, ${ }^{1}$ who found that the interclavicular midpoint was closely related to the true tracheal midpoint, though they did not relate either to the vertebral landmarks as we have.

The variation in the position of the clavicles is unlikely to be due to our technique as a standardised and consistent technique was used for all the infants. Rotation of the head and varying degrees of flexion and extension of the neck are known to alter the position of endotracheal tubes ${ }^{10}$ on chest radiographs, but there is no evidence to suggest that the position of the clavicles is affected. It is possible that the degree of inflation of the lungs might alter the position of the sternum relative to the back, but this is unlikely as most of our patients had the same disease, namely respiratory distress syndrome, and, hence, similar degrees of underinflation due to poor lung compliance.

In this study, the position of the carina was shown to be consistent, which is similar to the findings of Goodman et al in adults that the carina was between T5 and T7 in 92 of 100 chest radiographs. ${ }^{11}$ In all of our radiographs the carina was between T3 and T5 (see fig 1) and in $85 \%$ it was at either T3-4 or T4. In the group of 23 patients who had more than one chest radiograph, the variability ranged from zero to one intervertebral disc spaces, with an average of 0.5 intervertebral disc spaces (or 2.2 $\mathrm{mm}$ ). The constancy of the position of the carina makes it a good reference point, but its visibility is dependent on good technique. We had to reject three radiographs because the carina was not clearly visible, a rate of $3.57 \%$, though in the clinical setting the rate seems to be much higher.

We have also confirmed the findings of Goodman et al that the carina migrates caudally with age. ${ }^{11}$ At birth, the carina is situated close to T4 regardless of gestational age. Our technique is unlikely to be responsible for projection errors and we excluded combined chest and abdomen radiographs which might cause an upward displacement of the carina relative to the thoracic vertebrae if the beam was aimed at a point other than the sternal angle. There is no evidence to suggest that the position of the head alters the position of the carina. Because the carina moves cephalad on expiration, ${ }^{10}$ every effort was made to coincide the shooting of the film with peak inspiration by observing the movement of the infants' chest and the inspiratory phase of the ventilator breath.

Analysis of variance shows that the position of the clavicles was determined with significantly higher variation ( $F$ test $=5.39 ; p<0.01$ ) than the position of the carina. This makes the clavicles a poor reference for either clinical use or comparison studies.

We also measured the distance in centimetres from the carina to the clavicles and $\mathrm{T} 1$, and our findings confirm that the trachea increases in length as birth weight increases. Our results are consistent with those of Bednarek and Kuhns, ${ }^{1}$ who, using the interclavicular midpoint as a marker of the tracheal midpoint, showed that the distance from the carina to the interclavicular midpoint was 1.64 $\mathrm{cm}$ at less than 30 weeks', $2 \cdot 1 \mathrm{~cm}$ at $31-37$ weeks', and $2.4 \mathrm{~cm}$ at $38-40$ weeks' gestation. Our results were $1.8 \mathrm{~cm}, 2.4 \mathrm{~cm}$, and $3.2 \mathrm{~cm}$ for the same groups. The intrapatient variation ranged from zero to $1.2 \mathrm{~cm}$, with an average of $0.51 \mathrm{~cm}$. The greater variability in interclavicular midpoint seen in our patients may be related to chest radiograph selection as Bednarek and Kuhns ${ }^{1}$ used only chest radiographs with the 'head and clavicle in the neutral position', whereas we required only the head to be in the neutral position.

The distance from the carina to $T 1$ is $1.2 \mathrm{~cm}$ at $500 \mathrm{~g}$, and increases to $1.7 \mathrm{~cm}$ at $3600 \mathrm{~g}$. The intrapatient variation ranged from $0 \cdot 1$ to $0.6 \mathrm{~cm}$, with an average of $0.22 \mathrm{~cm}$. The body of $\mathrm{T} 1$ is the posterior marker of the thoracic inlet and has been suggested as a reference point by Heller and Cotton in their study of illuminated endotracheal tubes, though they did not actually refer to it in their results. ${ }^{2}$ Its position on chest radiograph should theoretically be fixed, but does it bear a constant relation to mobile structures, such as the carina? Our study suggests that it does.

Bednarek and Kuhns reported that the ideal position for the placement of an endotracheal tube in an infant would allow for the following: (a) $1.2 \mathrm{~cm}$ upward movement on head turning; (b) $0.5 \mathrm{~cm}$ descent on head flexion; and (c) a maximum distance of $2 \mathrm{~cm}$ above the carina itself to allow for movement of the carina during respiration. ${ }^{1}$ These figures apply only to full term infants. We have shown that $T 1$ fulfils these criteria and can be used at any gestational age as it makes allowances for changes in tracheal length in infants from 500 to $3600 \mathrm{~g}$. Its visibility makes it a superior marker to the carina, though in this study a good radiographic technique allowed the carina to be seen in all but three chest radiographs. The only disadvantage in using $T 1$ is related to its identification. It may sometimes be difficult to follow the first rib onto $T 1$; however, when the ribs are counted as described in 
this study, T12 can always be identified, even in the presence of cervical ribs, or absent ribs. We never disagreed when this method was used.

In summary, we have shown that the position of the clavicles on a chest radiograph is too variable to use them as a reference point for endotracheal tube placement, and that, though the carina and $\mathrm{T} 1$ bear a more constant relation to the desired tracheal midpoint, the visibility of $\mathrm{T} 1$ is superior, and its use would obviate the need to measure the distance from the carina. We therefore recommend that, in the neonatal period, the tip of the endotracheal tube should be positioned at the body of the first thoracic vertebra on chest radiographs, as this will ensure accurate placement within the trachea, and that T1 could be used as the only standard reference point for endotracheal tube location in preference to either the medial ends of the clavicles, the distance above the carina, or a combination of the two.
We thank Dr E Sauerbrei and Dr B MacMurray for reviewing the manuscript, and Dr M Lam, department of community medicine, Queen's University, for the statistical analysis.

1 Bednarek FJ, Kuhns LR. Endotracheal tube placement in infants determined by suprasternal palpation: a new technique. Pediatrics 1975; 56: 224-9.

2 Heller RM, Cotton RB. Early experience with illuminated endotracheal tubes in premature and term infants. endotracheal tubes in p

3 Slovis TL, Poland RL. Endotracheal tubes in neonates: sonographic positioning. Radiology 1986; 160: 262-3.

4 Blayney MP, Costello S, Perlman M, Lui K, Frank J. A new system for location of endotracheal tube in preterm and term infants. Pediatrics 1991; 87: 44-7.

5 Hauser GJ, Pollack MM, Sivit CJ, Taylor GA, Bulas DI, Guion CJ. Routine chest radiographs in pediatric intensive care: a prospective study. Pediatrics 1989; 83: 465-70.

6 Bloch EC, Ossey K, Ginsberg B. Tracheal intubation in children: a new method for assuring correct depth of tube placement. Anesth Analg 1988; 67: 590-2.

7 Halliday HL. McLure G, Reid M. Handbook of neonatal intensive care. 2nd Ed. London: Ballière Tindall, 1985: 65.

8 Fletcher BD, Morrison SC, Yulish BS. Diagnostic imaging. Neonatal-perinatal medicine. Diseases of the fetus and infant. 4th Ed. St Louis: Mosby, 1987: 183-213.

9 SAS Institute Inc. SAS/STAT user's guide, version 6. 4th Ed Vols 1 and 2. Cary, NC: SAS Institute.

10 Kuhns LR, Poznanski AK. Endotracheal tube position in the infant. $f$ Pediatr 1971; 78: 991-6.

11 Goodman LR, Conrardy PA, Laing F, et al. Radiographic evaluation of endotracheal tube position. $A \mathcal{Y} R$ 1976; 127: 433-4. 